\title{
ARTICLE
}

\section{Development of Embedded DAQ System for radiation monitoring using counting and integration methods.}

\author{
Yongseok HWANG ${ }^{1}$, H.J. KIM ${ }^{1 *}$, Sanghoon $\mathrm{LEE}^{1}$, Sunghwan $\mathrm{KIM}^{2}$ \\ ${ }^{1}$ Kyungpook National University, Daegu 702-701, Korea \\ ${ }^{2}$ Department of Radiation Science, Cheongju University, Cheongju 360-764, Korea
}

\begin{abstract}
This study has developed an embedded Data Acquisition (DAQ) system for the radiation monitoring using two different methods which are counting and integration. The counting method is useful to measure the detectors signal accurately when radiation enters the detector. But it becomes increasingly difficult to distinguish individual signals when the signal pulse is increased. On the other hand, the integration method is better at measuring the high flux signals, but it could be difficult to measure the low flux due to the electronics noise. Thus an embedded system was developed by using both methods based on the ATmega128 microcontroller and the charge integration chip. This system can be operated in both low and high radiation flux environments. The data is recorded into a personal computer with a linux operating system in real time through the serial connection. The data was analyzed with the C++based data analysis program, ROOT package. This embedded DAQ system was tested with test pulse. The developed system is portable and useful for flux monitoring in various radiation environments.
\end{abstract}

\section{KEY WORDS: ATMEGA128, AVR, AD7746, embedded, DAQ, radiation monitoring}

\section{Introduction}

This paper discusses the test of charge integration and counting embedded board for the proton beam monitoring. The goal is monitoring the proton beam flux by counting with a single crystal scintillator attached with photomultiplier tube (PMT) and charge integration with ion chamber. These electrical signals were measured by two distinct methods: single particle counting or integration of the signal current. The counting is to measure the number of particles from the scintillator, which is converted to voltage signals by PMT. With rising particle flux, the counting method becomes increasingly difficult to distinguish individual charge pulses and the number of unregistered events increases. The other method is charge integration to measure the currents in the ion chamber. The integrator is well suited in measuring the high flux signal currents, but the low flux measuring could be difficult due to the electronic noises of the detector [1]. Both counts method are employed using the ATmega128 microcontroller and the charge integration method using the DDC112 charge integration chip. The ATmega128 was selected for the embedded DAQ system since it is easy to make the firmware by using $\mathrm{C}$-code and easy to operate.

\section{Experiments}

\section{Embedded DAQ System Specification}

The embedded DAQ system contains two basic elements:

*H.J.KIM, Tel. +82-53-950-5323, Fax. +82-53-952-1739,

E-Mail; hongjoo@knu.ac.kr

(C) Atomic Energy Society of Japan the ATmega128 (8-bit microcontroller) [2] and DDC112 (dual current input 20-bit analog to digital converter) [3]. The ATmega128 has a 128-Kbytes reprogrammable flash memory for the firmware storage, 16-bit Timer/Counter unit allows accurate program execution timing, wave generation and signal timing measurement. Especially the counter input source pin (T1 input port) can be used as 16-bit Timer/Counter for the beam flux monitoring. However, due to the variation of the system clock frequency and duty cycle caused by oscillator source tolerances, it is recommended that maximum frequency of an external clock source is less than $\mathrm{clk}_{\mathrm{I} / \mathrm{O}}$ (system clock : $8 \mathrm{MHz}$ ) /2.5. Thus, was possible to record up to $3.2 \times 10^{6}$ particle $/ \mathrm{sec}$.

The DDC112 has a dual input, wide dynamic range, charge-digitizing analog to digital convertor with 20-bit resolution. Charge integration is continuous as each input uses two integrators so that while one is being digitized, the other is integrating. For each of its two inputs, the DDC112 combines current-to-voltage conversion, continuous integration, programmable full-scale range, external integration capacitors allow an additional user-settable fullscale range of up to $1000 \mathrm{pC}$. The value of the integrator's feedback capacitor, the integration period, and the reference voltage determine the positive full-scale $(+\mathrm{FS})$ range of the DDC112 is given by the following equations. The negative full-scale (-FS) range is approximately $0.4 \%$ of the positive full-scale range. The maximum measureable current is given by

$\mathrm{I}_{\mathrm{FS}}=(0.96) \cdot \mathrm{V}_{\mathrm{REF}} \cdot \mathrm{C}_{\mathrm{F}} / \mathrm{T}_{\mathrm{INT}}=$ Input range $(\mathrm{pC}) / \mathrm{T}_{\mathrm{INT}}$ 
, where $\mathrm{T}_{\mathrm{INT}}$ is the charge integration time. It has the range from $50 \mu \mathrm{s}$ to $1 \mathrm{~s}$ in non-continuous mode and from $500 \mu \mathrm{s}$ to $1 \mathrm{~s}$ in continuous mode. $\mathrm{C}_{\mathrm{F}}$ is the value of the integrator's feedback capacitor. The " 0.96 " factor allows the front end integrators to reach full scale to the ground. The $V_{R E F}$ is the external voltage reference which is used to reset the integration capacitors before an integration cycle begins.

Fig. 1 shows the schematic of the board and the diagram of the system. The signal was processed step by step, integration and counting. Counting data was written with taken time. So the counting rate can also be measured. Integration data have only information of the currents signal, the integration time was selected by the user.

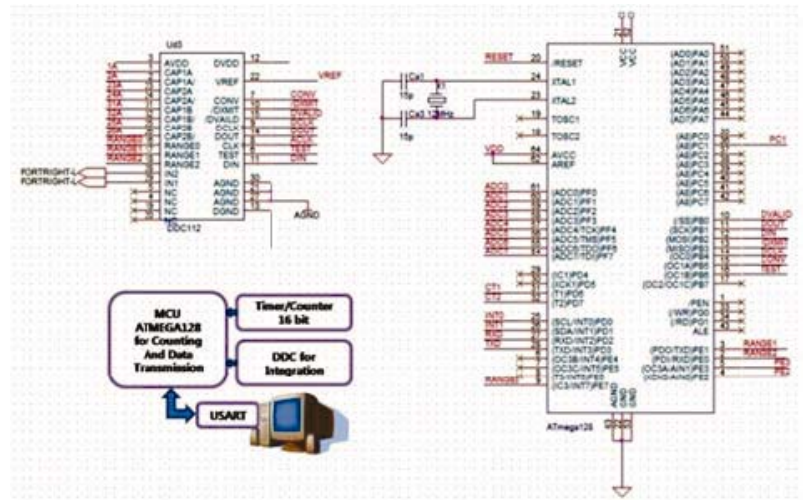

Fig. 1 Circuit and data flow diagram of the board.

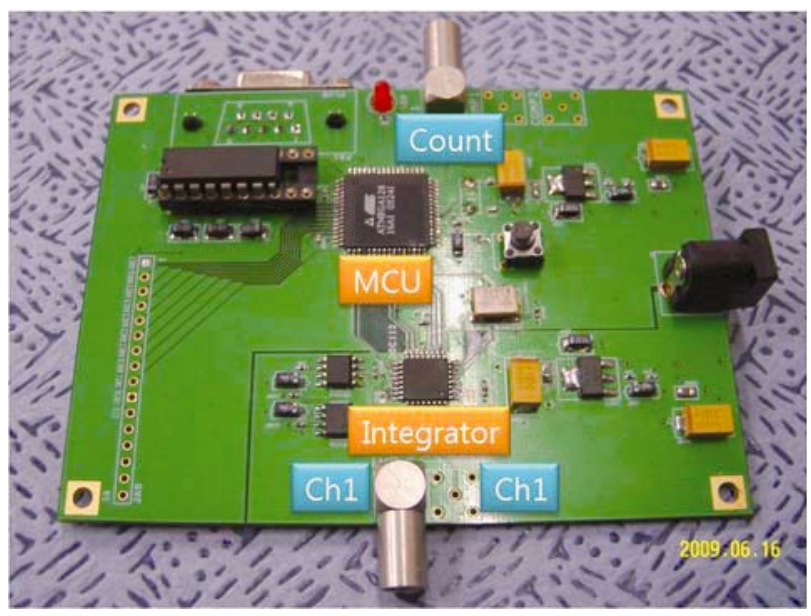

Fig. 2 ATmega128 DAQ board. It is consist of a two channel current integrator and a counter. The size of the board is $8 \mathrm{~cm} \times 10 \mathrm{~cm}$.

This board has two different measurements system for counting and integration. Fig. 3 shown the counting and integration information recorded into the personal computer with a linux operating system in real time through the serial connection. The data was analyzed with the $\mathrm{C}++$-based data analysis program, ROOT package [4]. Counting data shows the real time count and count rate in top of the window. The currents data was monitored in the middle and bottom window. The original data and data taking times were displayed on the terminal screen.

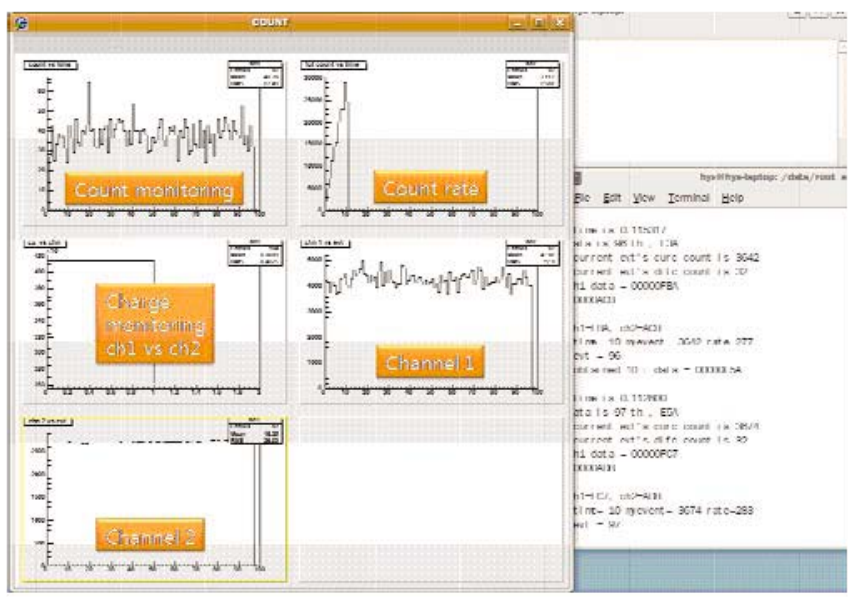

Fig. 3 Monitoring and data taking program.

\section{Prototype Counting System Test for Proton Beam Flux Monitoring}

A prototype counting board for testing the proton beam flux was made. This prototype board only contained the function of counting. The board was tested in the $50-\mathrm{MeV}$ proton beam test facility at the MC-50 cyclotron of Korea Institute of Radiological and Medical Sciences (KIRAMS) had been established by the PEFP (Proton Engineering Frontier Project) of the Korea Atomic Energy Research Institute (KAERI). This facility will be used for pilot studies of the PEFP, especially for studies using low-flux proton beams, $10^{4} \sim 10^{10} / \mathrm{cm}^{2} \mathrm{sec}$. The beam line is composed of a collimator, a vacuum tube for beam drift, bellows for easy alignment, a beam profile monitor (BPM), an exit window for an external beam, a phosphor screen, a scattering foil, a energy degrader, a target stage, an irradiation uniformity measurement system, a dose measurement system, an energy measurement system [5], etc. A $35-\mathrm{MeV}$ proton beam was used to measure the proton beam flux. Fig. 4 shows the experimental setup of the proton beam line.

The GSO crystal was prepared for the proton beam flux monitoring. A PMT (Hamamatsu Co.) with $1 \mathrm{~cm}$ diameter was attached to the GSO crystal scintillator for the light conversion to the photo-electron. This electron was fed into the preamplifier for the current signal conversion to the voltage signal. After amplification the signals were put into the discriminator (ORTEC 715) for conversion to the NIM (Nuclear Instrument Modules) logic signal, this NIM logic signal was fed to the gate generator (ORTEC GG8010) for conversion to the TTL (Transistor-Transistor Logic) signal [6]. This TTL signal has $50 \mathrm{~ns}$ pulse width that was fed into the embedded DAQ board that has a counting capability. Also, a beam flux monitoring program was running during the beam test so that the proton beam flux could be monitored in real time. Fig. 5 shows a schematic diagram of the experimental setup for the beam flux monitoring. 


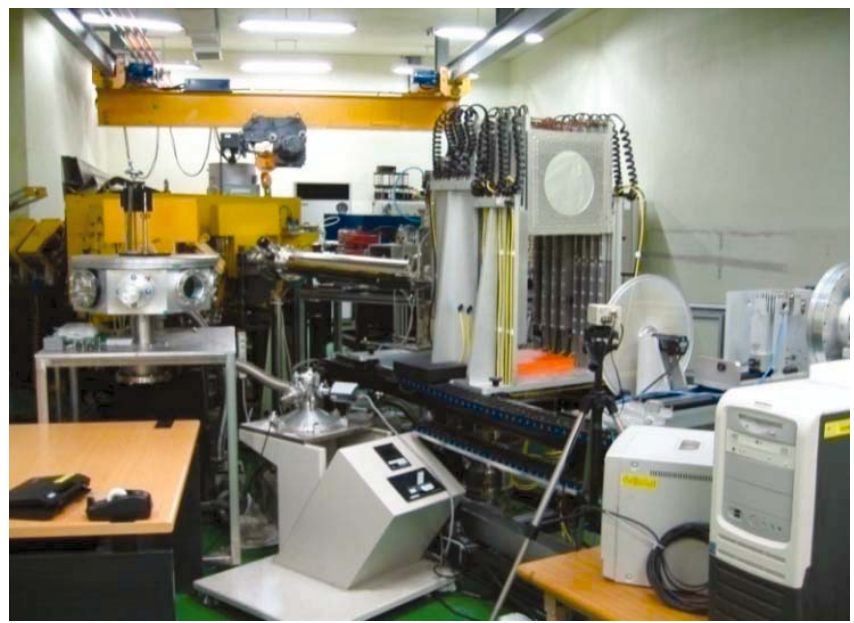

Fig. 4 A photograph of the proton beam line at KIRAMS.

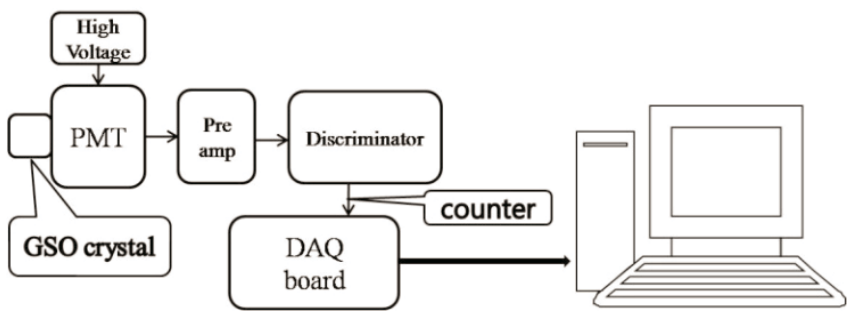

Fig. 5 Schematic diagram of the proton monitoring setup.

0.5- , 1-, 2-, 5-, and 10- nA beam current were measured in $35 \mathrm{MeV}$ proton beam flux as shown in Fig. 6. When the proton beam flux was more than $5 \times 10^{6}$ proton/sec and the pulse width less than 50ns, the present setup did not work, because the TTL signal was continuous in every system clock. In this case, the use of the integration method is the best option.

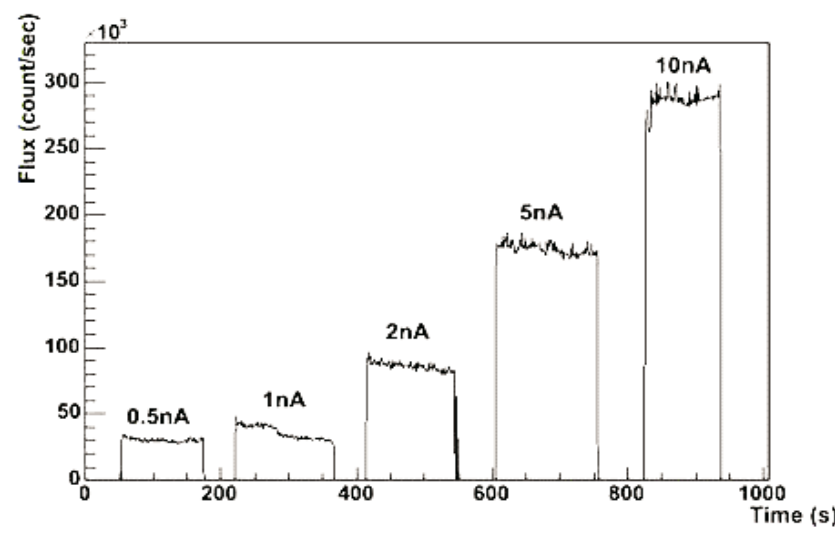

Fig. 6 Proton beam flux for 0.5-, 1-, 2-,5- and 10- nA beam currents.

\section{Test of Charge Integration board.}

Charge integration was measured by using a photodiode sensor (Hamamatsu Co. S3590) attached with a red LED. The LED generates light pulses from the function generator (Agilent 33250A). The current of photodiode was changed when different frequencies were applied. For example, the
$10 \mathrm{KHz}$ pulse with $10 \mu$ s pulse width was generated the current approximate $10 \mathrm{nA}$. It was also measured with the electrometer (KEITHLEY 6517). The linearity, dynamic range at noise performance was tested with current, the setup was as follows.

1) Linearity: Investigation of the integrator linearity with generated pulses from the photodiode. The integration time $3 \mathrm{~ms}$ and full charge range of $350 \mathrm{pC}$ was used. Fig. 7 shows applied current versus measured ADC using DDC112 coupled with the photodiode. Measured charge is linear with applied current.

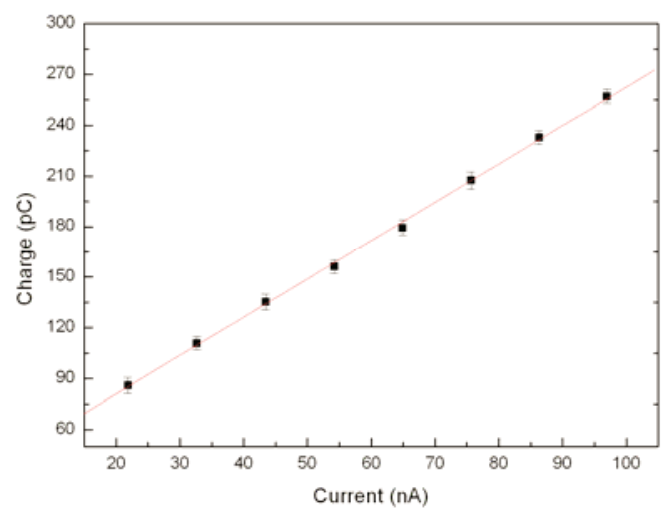

Fig. 7 Applied current versus measured ADC value.

2) Dynamic range: To change full dynamic range, either charge integrator capacitor or integration time can be changed. It can be measured the current full-scale from 50 pA to $7 \mu \mathrm{A}$.

3) Noise performance: Fig. 8 shows the background noise value when there is no current of the photodiode. The RMS value of background noise is measured to be $0.084 \mathrm{pC}$. It means the noise level is approximately $8 \mathrm{fC}$ when $50 \mathrm{pC}$ was chosen as the full dynamic range.

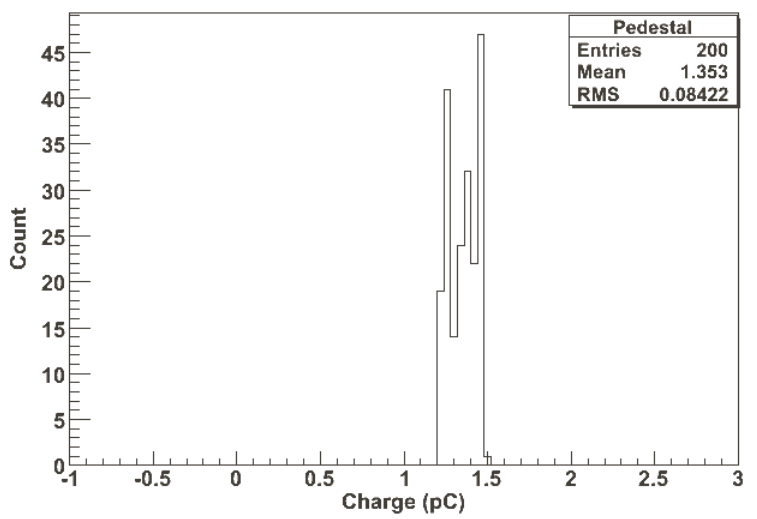

Fig. 8 Noise performance of the integrator.

4) Compare the Integration and Counting: The counting method is good for low flux measurements because the low flux is similar to the low frequency it is easy to measure 
with a microcontroller. But it will suffer from pileup when it is exposed to high flux. Then the analog signal couldn't be separated in each of the signal situations. The Integration mode exhibits a good for high flux signal measurements. But it is too sensitive so that it is difficult to measure low flux readings due to the electronic noise, likes a electromagnetic wave in the environments. Fig. 9 shown these effect of different methods. We have tested for currents readout up to $6 \mathrm{MHz}$ and the result was reasonable. However, the test for counting was working up to $4 \mathrm{MHz}$ and the pileup effect showed up above $4 \mathrm{MHz}$ frequency.
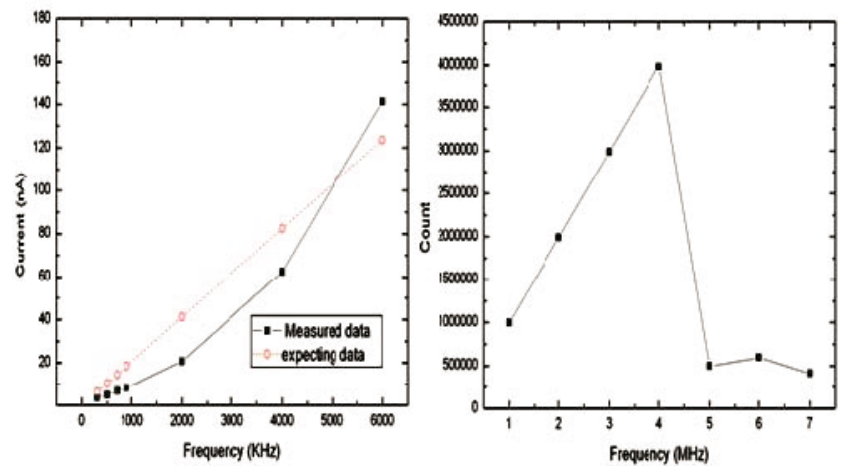

Fig. 9 Currents (left) and counting (right) readout with high frequency signals.

\section{Conclusion}

The embedded DAQ system consists of a counting and integrating signal processing method was developed for proton beam monitoring. The embedded DAQ system worked in both modes as the counter and the integrator. The proton beam flux was measured with a single crystal based detector for $35 \mathrm{MeV}$ proton beam from the MC-50 cyclotron at the Korea Institute of Radiological and Medical Science.

The proton beam flux for 0.5-, 1-, 2-, 5-, and 10- nA beam current was successfully monitored by using a counting method with an embedded process. The counter and the integrator was tested and shown to have the expected performance. TTL gate generator also are developed and tested, next version will add on. This system will be tested with proton beam, $x$-ray and gamma-ray by using an ion chamber or crystal scintillator in the near future.

\section{Acknowledgement}

This work was supported by the user program of Proton Engineering Frontier Project the KOMAC program.

\section{References}

1) Edgar Kraft, Peter Fischer, Michael Karagounis, Manuel Koch, Hans Kruger, Ivan Peric, Norbert Wermes, Christoph Herrmann, Augusto Nascetti, Michael Overdick and Walter Rutten, "Counting and integrating readout for direct conversion X-ray imaging concept, realization and first prototype measurements," Nuclear Science Symposium Conference Record, IEEE., 5[5],2761.(2005)

2) Duck-yong Yoon, “AVR ATMEGA128 MASTER”, Ohm publishing Co., Korea, 85 (2006).

3) BURR-BROWN from Texas Instrument co.LTD. URL:http://www.ti.com/

4) R. Brun and F. Rademakers, Nucl. Instr. Meth., A389, 111 (1997), URL: http://root.cern.ch.

5) K. R. Kim, B. S. Park, H. R. Lee, K. S. Kang, S. W. Kang and B. H. Choi, Proceedings of APAC (Asian Particle Accelerator Conference, Gyeongju), (2004).

6) J.H. SO, H.J. KIM, Heedong KANG, H. PARK, S. RYU, and S.W. Jung, Sihong DOH, Sunghwn KIM, Kyeryung KIM "Development of Lquid Scintillator System for Proton Flux Monitoring," J. Korean Phys. Soc., 50[4], 1506 (2007). 